\title{
Analysis of apical hook formation in Alaska pea with a 3-D clinostat and agravitropic mutant ageotropum
}

\author{
Kensuke Miyamoto $^{1}{ }^{*}$, Takahiro Yamasaki $^{2}$, Eiji Uheda ${ }^{3}$ and Junichi Ueda ${ }^{3}$ \\ ' Faculty of Liberal Arts and Sciences, Osaka Prefecture University, Sakai, Osaka, Japan \\ 2 Faculty of Science, Osaka Prefecture University, Sakai, Osaka, Japan \\ ${ }^{3}$ Graduate School of Science, Osaka Prefecture University, Sakai, Osaka, Japan
}

Edited by:

Tohru Hashimoto, Uozaki Life Science Laboratory, Japan

Reviewed by:

John Z. Kiss, The University of Mississippi, USA

Karl H. Hasenstein, University of

Louisiana at Lafayette, USA

\section{*Correspondence:}

Kensuke Miyamoto, Faculty of Liberal Arts and Sciences, Osaka Prefecture University, 1-1 Gakuen-cho, Naka-ku, Sakai, Osaka 599-8531, Japan e-mail:miyamoto@las.osakafu-u.ac.jp
The formation of the apical hook in dicotyledonous seedlings is believed to be effected by gravity in the dark. However, this notion is mostly based on experiments with the hook formed on the hypocotyl, and no detailed studies are available with the developmental manners of the hook, particularly of the epicotyl hook. The present study aims at clarifying the dynamics of hook formation including the possible involvement of gravity. Time-course studies with normal Alaska pea (Pisum sativum L., cv. Alaska) and an agravitropic pea mutant, ageotropum, under the 1-g conditions and on a 3-D clinostat revealed that (1) the apical hook of the epicotyl forms by the development of the arc-shaped plumule of the embryo existing in the non-germinated seed. The process of formation consists of two stages: development and partial opening, which are controlled by some intrinsic property of the plumule, but not gravity. Approximately when the epicotyl emerges from the seed coat, the hook is established in both pea varieties. In Alaska the established hook is sustained or enhanced by gravity, resulting in a delay of hook opening compared with on a clinostat, which might give an incorrect idea that gravity causes hook formation. (2) During the hook development and opening processes the original plumular arc holds its orientation unchanged to be an established hook, which, therefore, is at the same side of the epicotyl axis as the cotyledons. This is true for both Alaska and ageotropum under 1-g conditions as well as on the clinostat, supporting finding (1). (3) Application of auxin polar transport inhibitors, hydroxyfluorenecarboxylic acid, naphthylphthalamic acid, and triiodobenzoic acid, suppressed the curvature of hook by equal extents in Alaska as well as ageotropum, suggesting that the hook development involves auxin polar transport probably asymmetrically distributed across the plumular axis by some intrinsic property of the plumule not directly related with gravity action.

Keywords: ageotropum, apical hook, auxin polar transport, clinostat, epicotyl bending, microgravity, Pisum sativum

\section{INTRODUCTION}

The apical hook is the arc-shaped transient structure formed in seed germination process on top of the hypocotyl or epicotyl of dicotyledonous seedlings. It is believed that, when seeds germinate in the field, the apical hook is formed in the dark in soil and opens in response to light near the surface of soil, thus plays a role to protect the fragile apical meristem from possible injuries when passing through the soil (Taiz and Zeiger, 2010). When the hook is formed in the dark, that gravity plays a key role was shown in sunflower, cress and cucumber (MacDonald et al., 1983; Takahashi and Suge, 1988) by means of a clinostat or other means.

The advent of experiments in a spacecraft or a space station made it possible to compare the growth and development of plants under 1- $g$ conditions on the earth with those under the microgravity ones in space to learn the effects of gravity (see Halstead and Dutcher, 1987; Hoson and Soga, 2003; Paul et al., 2013). In the STS-95 space experiments, NASA, the present authors also joined, and discovered that Alaska pea seedlings grown in the dark in space developed the epicotyl in an oblique upward direction away from the cotyledons and elected the root also in an upward direction asymmetric to the epicotyl. Besides the peculiar morphology of the shoot and root, the apical hook was also found to be markedly reduced in curvature (Ueda et al., 1999, 2000). A similar abnormal growth pattern of a seedling was observed to occur in an agravitropic pea mutant, ageotropum, under 1- $g$ conditions in the dark (Schöldéen and Burström, 1960; Olsen and Iversen, 1980a,b; Strudwick etal., 1997). The anomalous shape occurs not at random but uniformly in the majority of seedlings tested, leading to the idea that it is regulated by some intrinsic property of the seedlings, which is manifested first when the action of gravity is removed. This concept was already proposed by Pfeffer (1904) as automorphosis (Eigenrichtung; reviewed by Stanković et al., 1998) and served for explaining the establishment of intracellular polarity and determination of the growth direction in space (Volkmann etal., 1986) or on a clinostat (Hoson et al., 1992, 1996, 1997). 
As stated in the first paragraph, most experiments on the apical hook formation used epigeal plants as materials, the hypocotyl of which raises the hook upto near the soil surface; while in hypogeal plants no detailed studies on the apical hook formation are available. Furthermore, most studies were concentrated on the hook already established on an elongated hypocotyl, but rarely dealt with the process of hook development. The findings that Alaska pea seedlings formed the apical hook in space or on a clinostat, even if less developed, suggest that development of the apical hook may be caused by some intrinsic property (automorphosis) of pea seedlings besides gravity. In such background the present study aims to clarify how the apical hook develops, and how the intrinsic property and/or gravity are involved in the hook development. To achieve the aims the whole process of hook development is followed under the $1-g$ conditions in comparison with that obtained on a 3-D clinostat. The same experiments are carried out with ageotropum, to provide another control in addition to the one on a clinostat. Finally, the possible involvement of auxin polar transport is examined with relevant inhibitors. The above-planned analyses of the apical hook of pea seedlings are to increase understanding of apical hook formation in the hypogeal seedlings which has seldom been investigated.

\section{MATERIALS AND METHODS \\ PLANT MATERIALS AND CULTURE}

Two kinds of pea plants, Pisum sativum L., cv. Alaska and an agravitropic mutant, ageotropum, were used. Seeds of Alaska were purchased from Watanabe Seeds, Misato, Miyagi, Japan and seeds of ageotropum were propagated in the experimental field of the laboratory from the seeds kindly supplied by Prof. Hideyuki Takahashi, Tohoku University, Sendai, Japan. As seed bed, rock wool blocks, $9 \mathrm{~cm} \times 4.8 \mathrm{~cm} \times 1.5 \mathrm{~cm}$, cut out from a large sheet of rock wool (Chibikko Ace Mat, Nippon Rockwool Co. Ltd., Tokyo, Japan) were individually placed in acrylic resin boxes $(9 \mathrm{~cm} \times 4.8 \times \mathrm{cm} \times 5.8 \mathrm{~cm})$ of an exactly fitting size. For ventilation each box had four holes, $1 \mathrm{~cm}$ in diameter, in the ceiling, and the holes were covered with hydrophobic fluoropore membrane (MilliSeal, Millipore, Merck). On the seed beds so prepared, 12 seeds each were set in the manner that a whole seed was buried beneath the block surface, and the seed axis (the line to connect the plumular axis and radicle) was normal to the upper surface of the block. After supplied with $40 \mathrm{ml}$ water, each box was placed in a zipper-locked bag and kept at $23.5^{\circ} \mathrm{C}$ in the dark under $1-g$ conditions or on a three-dimensional clinostat (3-D clinostat).

\section{3-D CLINOSTAT}

It was manufactured by Nihon Ikakikai, Ltd., Osaka, Japan according to the original design by Hoson et al. $(1988,1992)$ and its operation was controlled with a rotation control system (Model CL-CS1, Minamide System Engineering, Ltd., Osaka, Japan). The clinostat system was composed of a clinostat within which another clinostat was equipped, and both clinostats were rotated independently at a variable rate up to $2 \mathrm{rpm}$, changing the rate and direction of rotation so that the gravity action integrated in all directions was null.

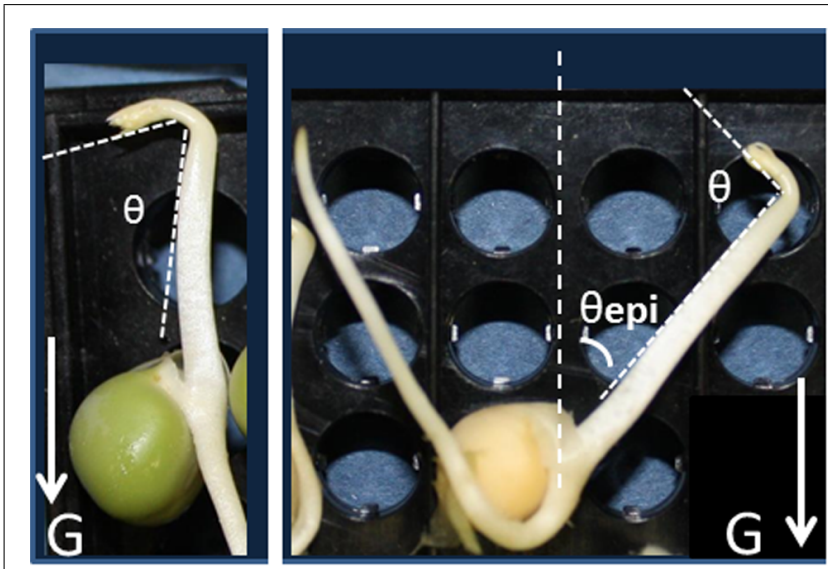

Alaska

ageotropum

FIGURE 1 | Pea seedlings, cv. Alaska and agravitropic mutant, ageotropum, grown under $1-\mathrm{g}$ in the dark at $23.5^{\circ} \mathrm{C}$ for $84 \mathrm{~h}$ asw (after supplying water to dry seeds), and the definition of hook angle $(\theta)$ and epicotyl bending $\left(\boldsymbol{\theta}_{\text {epi }}\right)$. The $\mathrm{G}$ arrows indicate the direction of gravity. In ageotropum the epicotyl extends in the direction of about $40^{\circ}$ away from the seed axis, and the root elongates in the symmetric direction to the epicotyl. Note that the apical hook bends on the same side of the epicotyl as the cotyledons in both pea varieties.

\section{DETERMINATION OF APICAL HOOK AND EPICOTYL BENDING}

Seedlings grown as above were harvested and photographed at the time(s) indicated, and the angles of apical hook and epicotyl bending were determined with a protractor on enlarged photographs. As shown in Figure 1, the apical hook angle represents the angle formed by the straight parts above the apical hook and the subapical epicotyl part mainly consisting of the elongation zone, and the epicotyl bending, the angle between the seed axis and the lower straight part of the epicotyl. To find the seed axis easily, a needle was stood in the gap of the cotyledons prior to being photographed (Figure 7).

\section{LOCALIZATION OF AMYLOPLASTS}

Seedlings standing on a growth bed served for staining. On one side of the epicotyl a longitudinal incision spanning from the lower end of the apical hook to about $10 \mathrm{~mm}$ below was made, and a drop of $\mathrm{I}_{2}-\mathrm{KI}$ solution was applied to the incision and allowed to diffuse into tissues. After $5 \mathrm{~min}$ when the tissues were fixed with the staining reagent, the epicotyl was sliced into about $100 \mu \mathrm{m}$ thick pieces with a razor blade by hand and photographed under a lightmicroscope (Olympus BH2, Tokyo). The method was according to Scott (1988).

\section{APPLICATION OF INHIBITORS}

The tested inhibitors were auxin polar transport inhibitors, 9-hydroxyfluorene-9-carboxylic acid (HFCA), N-(1-naphthyl) phthalamic acid (NPA), and 2,3,5-triiodobenzoic acid (TIBA). They were purchased from Sigma (St. Louis, MO, USA) or Tokyo Kasei Kogyo Ltd. (Tokyo, Japan) and used without further purification. They were individually dissolved in water at $10 \mu \mathrm{M}$, and 
applied, instead of plain water for starting germination, to dry rock wool where dry seeds had already been buried.

\section{RESULTS}

\section{AGRAVITROPIC MUTANT AGEOTROPUM MIMICS ALASKA PEA SEEDLINGS GROWN UNDER MICROGRAVITY CONDITIONS}

Under the microgravity in space and a simulated microgravity on the 3-D clinostat in the dark, etiolated pea seedlings of normal cultivar Alaska represented abnormal growth and morphology, i.e., the epicotyl bearing the partially opened apical hook grew in the oblique direction deviated by about $40^{\circ}$ away from the cotyledons and the root elongated in the oblique upward direction symmetric to the epicotyl. In order to learn how seedlings of the agravitropic pea mutant ageotropum grown under $1-g$ conditions simulates such abnormal growth and morphology that Alaska seedlings showed in space or on a clinostat, ageotropum seedlings were grown in the dark under 1- $g$ conditions and their growth and morphology were followed in comparison with Alaska seedlings during the germinating process for $96 \mathrm{~h}$ asw (after supplying water to dry seeds; Figures 1 and 2 ).

Seedlings of both varieties were harvested at intervals and photographed to collect data. Ageotropum seedlings showed abnormal orientation of the root and shoot, and reduced hook development, all of which were similar to those of Alaska observed under the microgravity conditions in space (Ueda et al., 1999, 2000) and on a 3-D clinostat (Miyamoto et al., 2005a,b, 2007). The epicotyl bending in ageotropum under $1-g$ conditions appeared already at $48 \mathrm{~h}$ asw when the epicotyl began to elongate and was maintained at least until $96 \mathrm{~h}$ asw (Figure 3). Furthermore it was not affected by rotation on a $3-\mathrm{D}$ clinostat. Thus, ageotropum seedlings were confirmed to be non-responsive to gravity and mimic Alaska seedlings grown under microgravity conditions, providing a criterion for inferring the gravity-related responses in apical hook development.

Fresh, iodine-stained longitudinal sections revealed that ageotropum seedlings appeared to have a normal content of amyloplasts which sedimented in the direction of gravity as observed

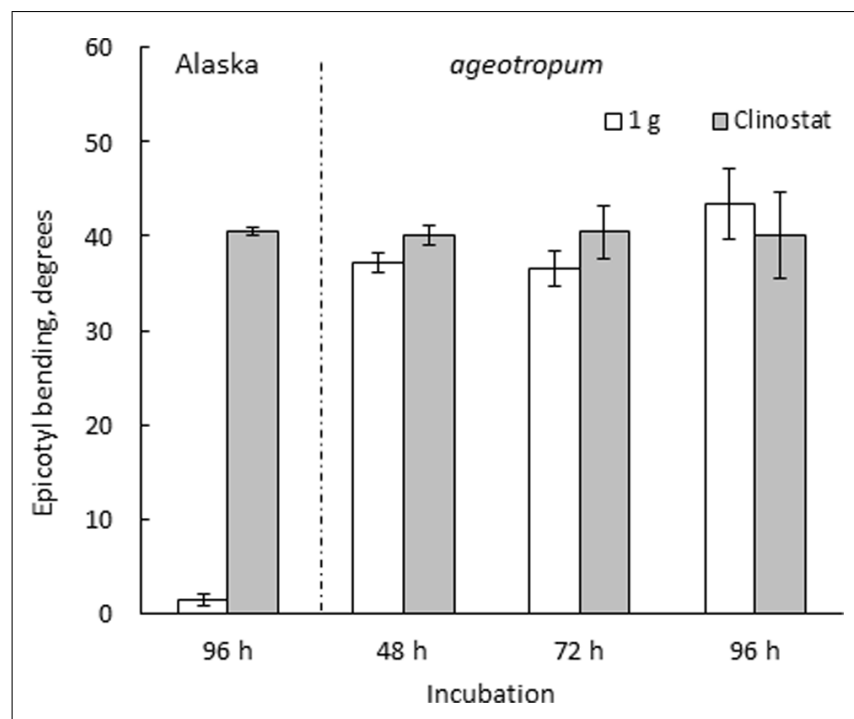

FIGURE 3 | Effects of 3-D clinostat rotation on the epicotyl bending in Alaska and ageotropum seedlings. Data bars: means with standard errors $(n=10)$; time: $\mathrm{h}$ asw.

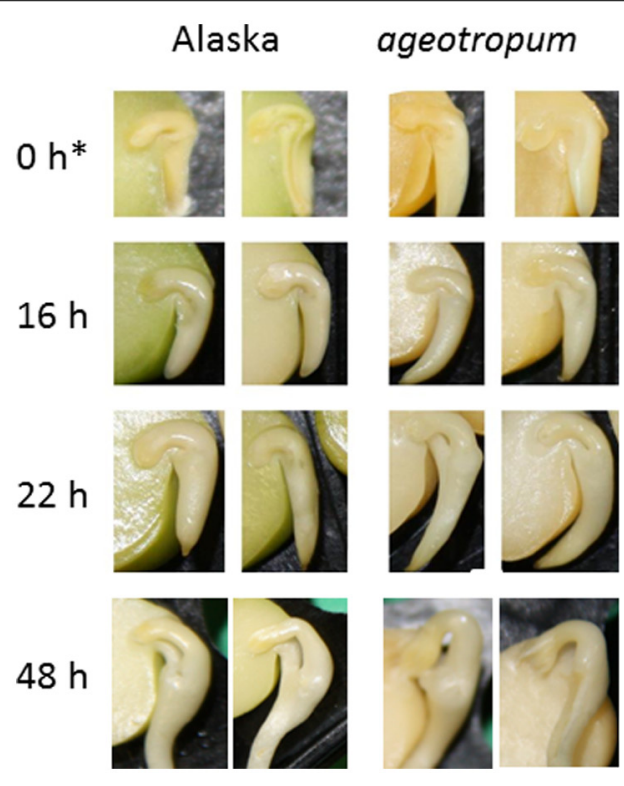

FIGURE 2 | Kinetics of the apical hook development in Alaska and ageotropum pea seedlings grown under $\mathbf{1 - g}$ conditions. Photographs were taken at the time points shown in $\mathrm{h}$ asw. To show the inside, the cotyledon at the front side was removed prior to photographing. Photos at $0 \mathrm{~h}^{*}$ show seeds imbibed in water in a refrigerator for $1 \mathrm{~h}$ to

\section{Alaska}

\section{ageotropum}

$72 \mathrm{~h}$

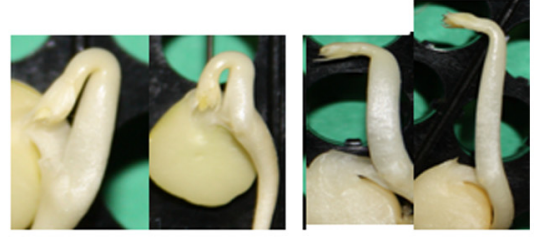

$96 \mathrm{~h}$

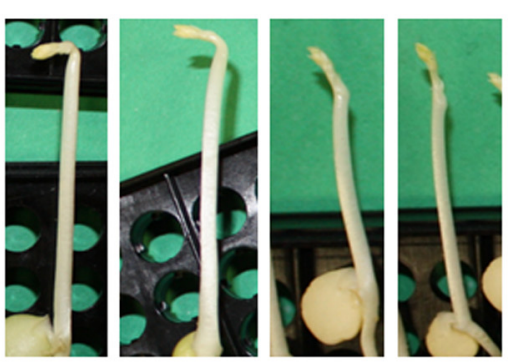

facilitate dissection. Although the epicotyl of ageotropum extended obliquely under $1-g$ conditions as well, the photos at $72 \mathrm{~h}$ and $96 \mathrm{~h}$ asw are arranged so that the epicotyl parallels that of Alaska for the convenience of comparing the hooks between the two varieties. Time is in $\mathrm{h}$ asw. 


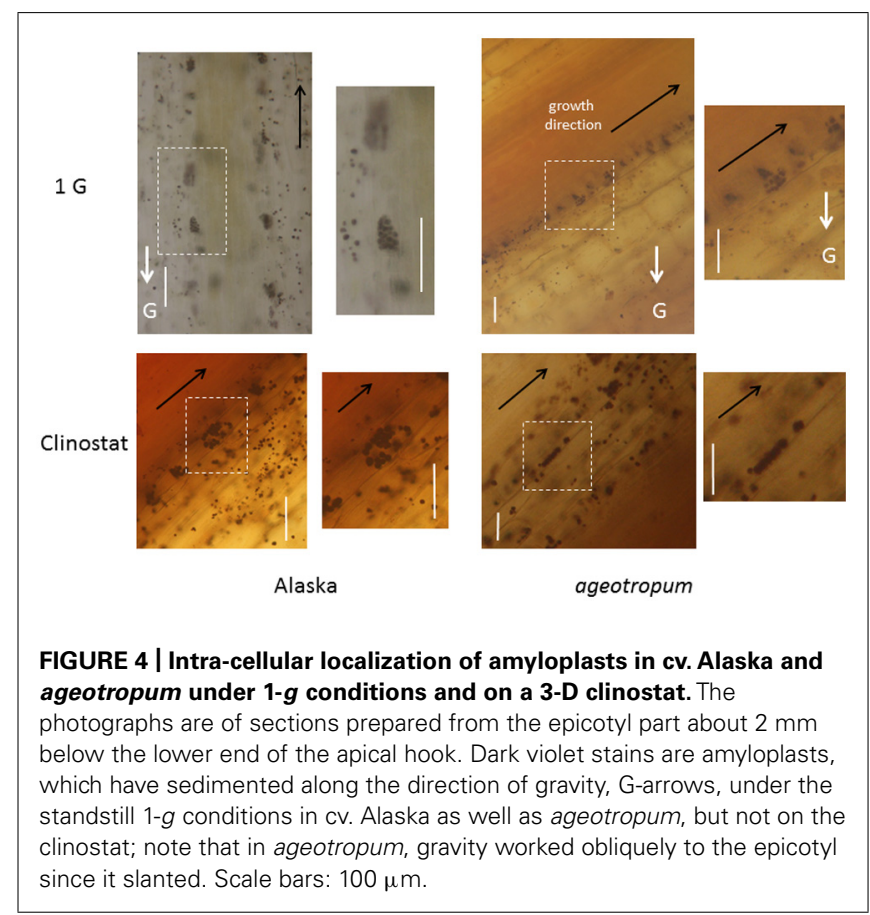

in Alaska seedlings (Figure 4). The result is similar to that reported with the root by Olsen and Iversen (1980b). The microscopic observation suggests that the amyloplasts and their sedimentation in epicotyl of ageotropum are normal, but its gravity perception/transduction system is considered to be disturbed in a step(s) other than amyloplast sedimentation.

\section{DEVELOPMENT OF APICAL HOOK IN ALASKA AND AGEOTROPUM PEAS UNDER 1-g CONDITIONS}

In Alaska pea, the arc-shaped plumule of embryo having an angle of about $90^{\circ}$ has already been formed in the embryo in dry seeds $\left(0 h^{*}\right.$ Figure 2$)$. Kinetic observation under $1-g$ conditions revealed that the apical hook was derived from the arc of plumule. As the epicotyl grew, the arc also developed intensifying its curvature, i.e., decreasing the angle of arc, by faster growth on the distal side to the cotyledons (outer side) than on the proximal side (inner side) from 22 to $48 \mathrm{~h}$ asw (Figure 2). Accordingly, the apical hook bent on the same side of the epicotyl as the cotyledons, or geometrically expressed the apical hook and the cotyledons shared a plane containing the epicotyl axis. A maximal curvature of the hook was reached when the epicotyl was ca. 5 10 $\mathrm{mm}$ long (72 h asw; Figures 2 and 5). Then, as the epicotyl elongated further, hook angle increased, i.e., hook opened partially even in the dark under $1-g$ conditions.

In ageotropum, an arc-shaped structure of plumule of the embryo in dry seeds gave rise to the apical hook similarly to the case in Alaska up to $48 \mathrm{~h}$ asw (Figures 2, 5, and 6). Subsequently, however, the hook shifted into the opening phase without such sustention or enhancement of hook as observed in Alaska. The sustention or enhancement of the hook found from 48 to $72 \mathrm{~h}$ asw is characteristic to the hook development in Alaska. Figure 2 shows, however, that Alaska seedlings developed the

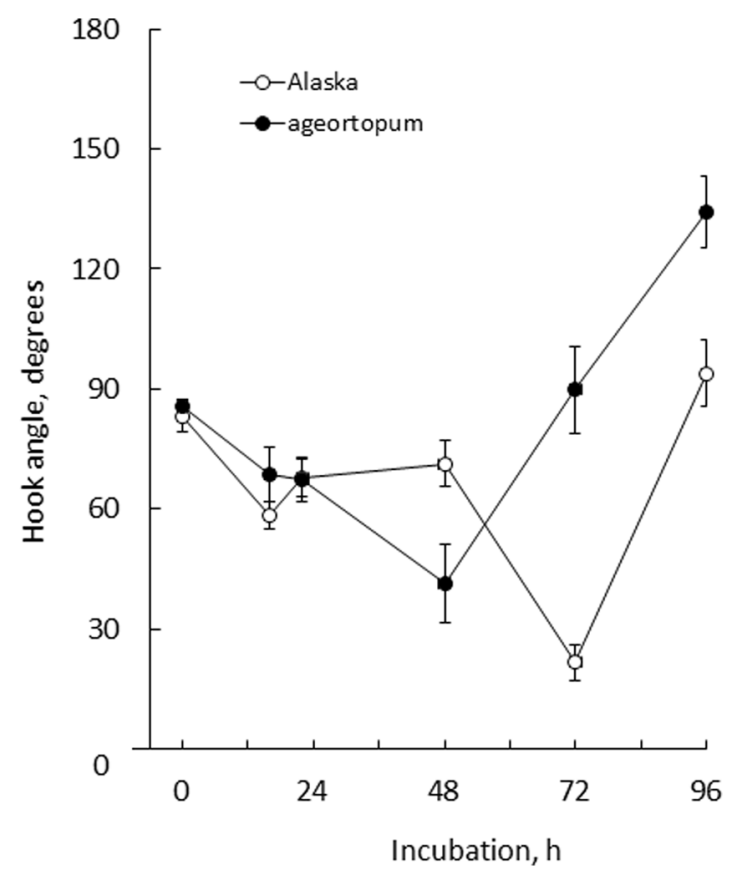

FIGURE 5 | Kinetics of the development of apical hooks in Alaska and ageotropum seedlings under $\mathbf{1 - g}$ conditions. Data points: the means with standard errors $(n=10)$; time: h asw.

hook slightly slower than ageotropum. Hence one might think that the delayed hook development of Alaska may have reflected to the difference in hook angle between Alaska and ageotropum 48$72 \mathrm{~h}$ asw, but this possibility will be removed by the subsequent experiments.

\section{APICAL HOOK DEVELOPMENT ON A 3-D CLINOSTAT}

The developmental path of the apical hook in Alaska was followed on the clinostat under $1-g$ conditions from 0 to $96 \mathrm{~h}$ asw in comparison with that observed under standstill $1-g$ conditions. In parallel, a similar experiment was also performed on ageotropum (Figure 6). In Alaska, until the hook was established at $72 \mathrm{~h}$ asw, no significant effect of clinostat rotation was noticed. In subsequent $24 \mathrm{~h}$, however, the established hook reduced its curvature markedly on the clinostat, whereas under the standstill conditions it maintained its sharp angle of arc. In ageotropum, by contrast, no significant effect of the clinostat was observed, as was expected from its non-responsiveness to gravity. Thus, the sustention of the curvature of the established apical hook observed in Alaska under $1-g$ conditions from 48 to $72 \mathrm{~h}$ is inferred due to gravity.

\section{EFFECT OF AUXIN POLAR TRANSPORT INHIBITORS}

In order to see if the apical hook development involves auxin transport, three inhibitors of auxin polar transport, TIBA, NPA, and HFCA, were individually tested on seedlings of cv. Alaska and ageotropum under $1-g$ conditions. Each inhibitor solution at $10 \mu \mathrm{M}$ was supplied to substrata in which seeds had been set and the results were determined after 96 h. For control, plain 

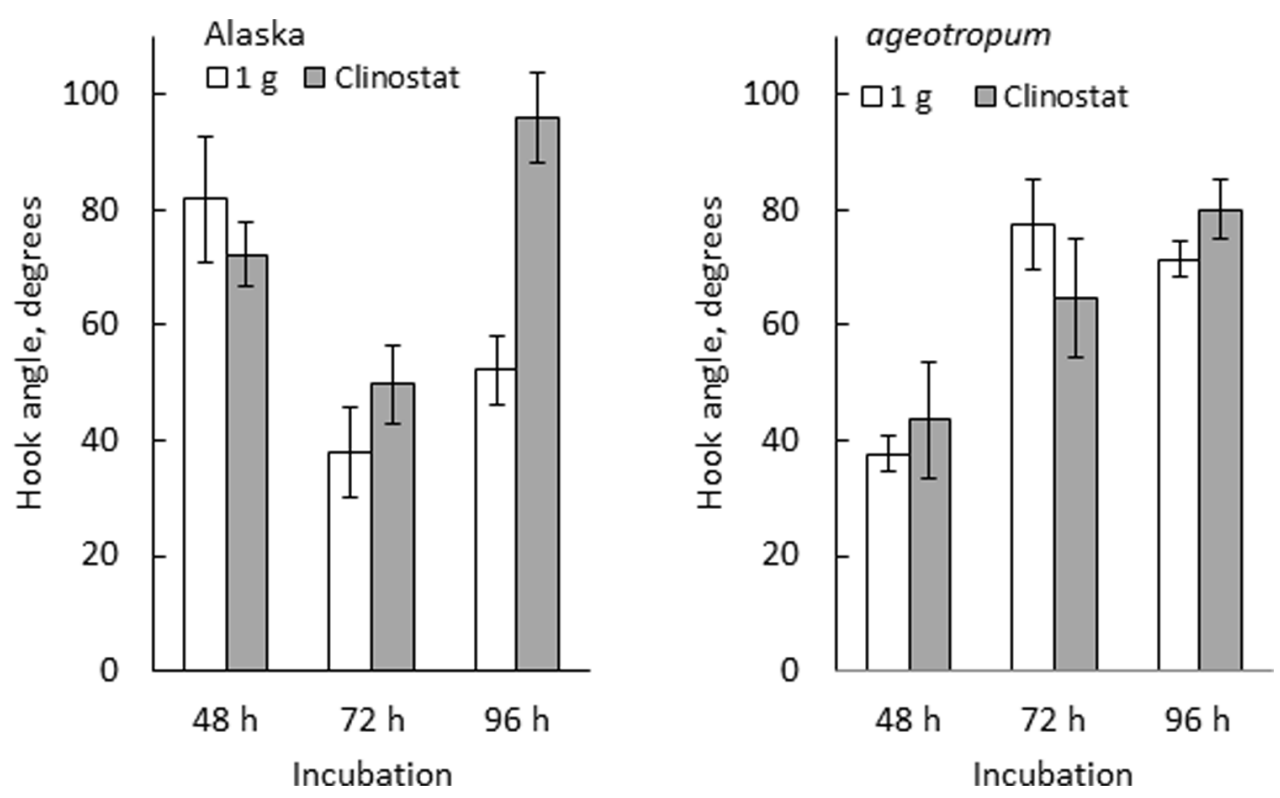

FIGURE 6 | Effects of clinostat rotation on the hook development in Alaska and ageotropum. Data bars: the means with standard errors ( $n=8-10$ ); time: h asw.

water was given. Fortunately, these inhibitors at the concentration used did neither affect seed germination, nor epicotyl elongation. As reported by Miyamoto et al. (2005b), the inhibitors caused epicotyl bending in cv. Alaska to the extent of $80 \%$ of plain water control on a clinostat (Figure 7), indicating that the inhibitor treatments were effective in inducing the epicotyl bending.

In addition, the same treatments reduced the extent of apical hook, i.e., opened the hook in Alaska nearly to the extent of the hook observed on the clinostat (Figure 7). Interestingly, the hook of ageotropum seedlings also was caused to open by percentages similar to those observed in Alaska (Figure 8). These findings indicate that auxin polar transport is involved in hook development and maintenance of the apical hook in Alaska as well as ageotropum. Being equally effective in both varieties of pea seedlings suggests that auxin polar transport controls hook development caused by the intrinsic property of seedlings independently of gravity, but it is not clear if the gravity-controlled phase of hook development, i.e., the enhancement and/or maintenance of the hook by gravity is also the case.

\section{DISCUSSION}

In the present study, the role of gravity in the formation and development of the epicotyl apical hook of dark-grown pea seedlings was examined by rotation on a $3-\mathrm{D}$ clinostat and comparison with the agravitropic pea mutant, ageotropum. The clinostat is designed and rotated at an appropriate rate so that the test plants mounted are uniformly subjected to gravity in all directions, hence integrated gravity of null (Hoson et al., 1992, 1996, 1997). Tests with the growth and development of seedlings of several species including pea have shown that the clinostat mimics the microgravity conditions in space (Kraft et al., 2000; Hoson and Soga, 2003). In fact, the shape of Alaska pea seedlings on the clinostat in the dark, the slanted epicotyl shown in Figure 7 for example, is similar to that observed in space (Ueda et al., 1999, 2000). The shape of ageotropum seedlings grown under $1-g$ conditions in the dark also is similar to that of Alaska obtained in space (Figures 1 and 6, Ueda et al., 1999, 2000). Thus, the use of the 3-D clinostat and ageotropum mutant is sufficiently qualified methods to examine the role of gravity on the development of the apical hook of pea seedlings on the ground.

The apical hook of pea seedlings is formed by the development of the arc-shaped plumule of embryo in the dark, accompanied by elongation of the epicotyl (Figure 2). Its formation process may be divided into two stages: development and partial opening. At the former stage the arc-shaped plumule develops to establish the hook, intensifying the curvature of arc, and at the latter stage the established hook opens partially even in the dark. It is noteworthy that both formation stages of the apical hook can take place independently of gravity, therefore do not require gravity, as shown by the experiments on a clinostat as well as with ageotropum (Figure 6). Under the $1-g$ conditions the established hook of cv. Alaska is sustained or enhanced before starting to partially open, therefore delayed to open compared with ones on a clinostat or ageotropum (Figures 2, 5, and 6). If judged at the latter stage, 72-96 h asw when the hook is established and the epicotyl starts vigorous growth (Figures 2 and 6), the hook formation might be recognized to be caused by gravity, but it is not correct. Gravity only enhanced or sustained the hook developed by the intrinsic nature of the plumule. Whether gravity works for an early limited period of or throughout the opening stage is not clear from the results obtained in 


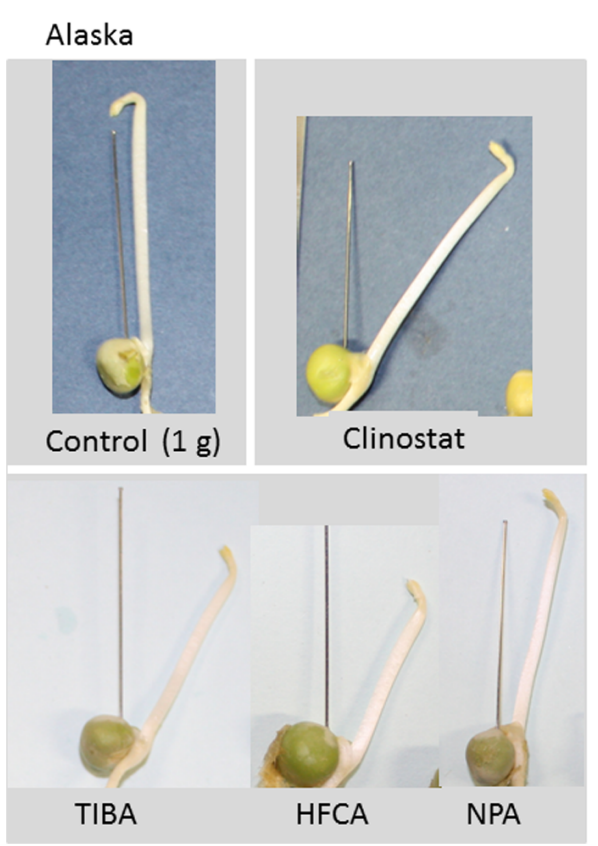

FIGURE 7 | Effects of auxin polar transport inhibitors on the hook development and the epicotyl bending in Alaska and ageotropum. Aqueous solutions $(10 \mu \mathrm{M})$ of inhibitors were individually added to the rock wool blocks embedding dry seeds, and seedlings were grown under 1-g

\section{ageotropum}

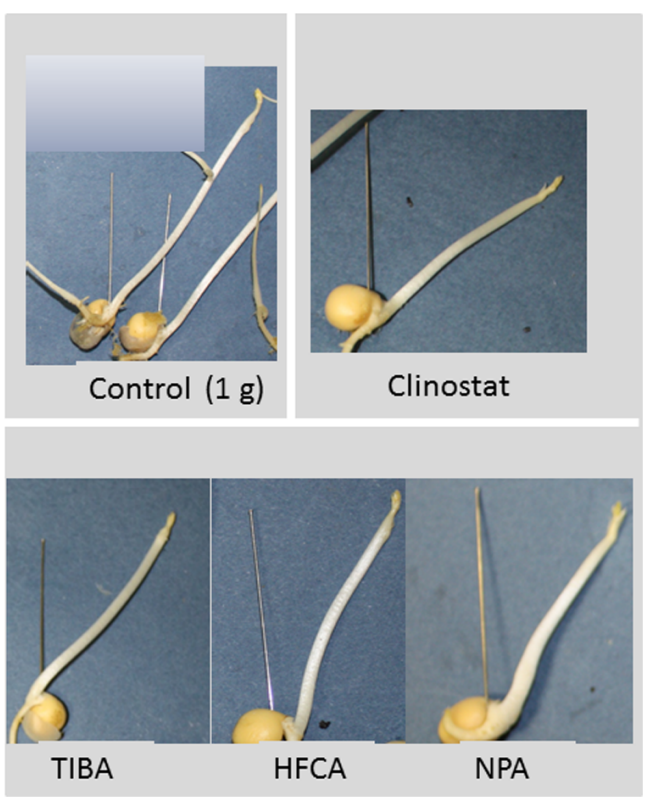

conditions for $96 \mathrm{~h}$ asw. Plain water controls were grown under 1-g conditions and also on the 3-D clinostat. To indicate the seed axis the needle was set up in the gap between the cotyledons prior to photographing.

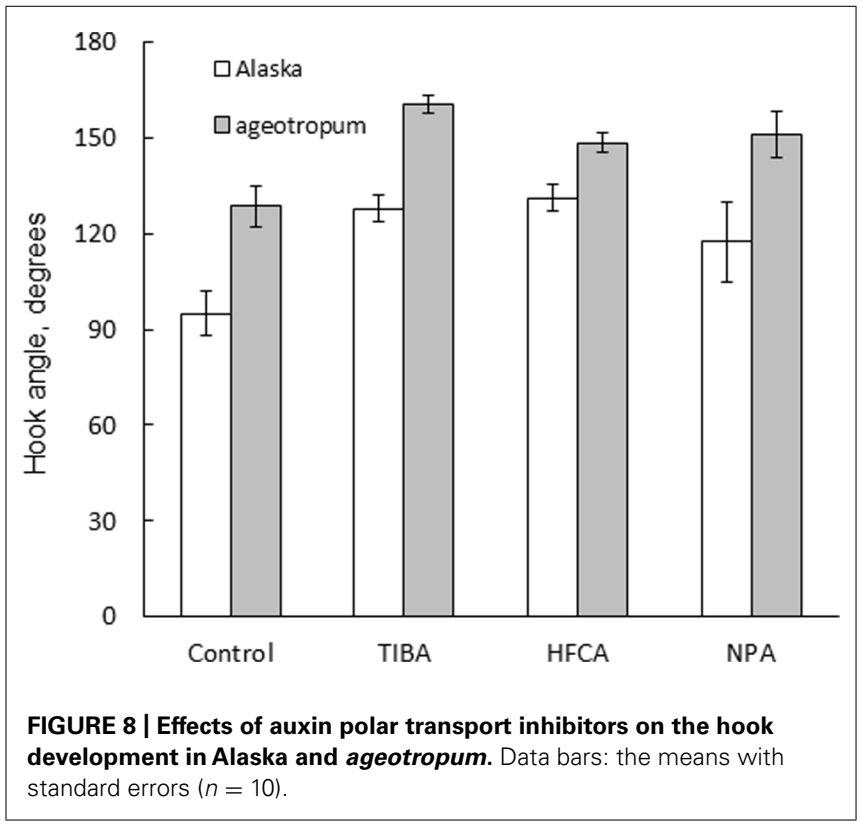

the present studies. In any case, at least in pea seedlings the hook formation is due to some intrinsic property of the embryo plumule, and gravity is only to sustain or enhance the established hook.

Certainly the hypocotyl of sunflower seedling placed in a horizontal position formed its apical hook in response to gravity, and cress seedlings rotated on a clinostat formed no hook
(MacDonald etal., 1983). Persimmon seeds sown in various directions produced all the downward-curved hook, except for seeds placed vertically with the micropyle end down, where the hypocotyl raised the seed part straight up without forming the apical hook until the hypocotyl received a diverged gravity owing to circumnutation of the hypocotyl top (Shichijo and Hashimoto, 2013). Thus, apical hook formation in the hypocotyl of several epigeal plants is caused by gravity. In hypogeal plants, on the other hand, to determine whether the manner of hook formation found in the present studies is the characteristic of epicotyl hooks must await accumulation of data with other hypogeal plants.

The hook development of Arabidopsis seedlings has been differentiated into three stages: formation, maintenance, and opening (Raz and Ecker, 1999; Vandenbussche et al., 2010; Žádníková et al., 2010). However, relating the stages of peas to those of Arabidopsis is difficult at present. Noteworthy is that both cases have the stage where the hook opens even in the dark.

All of the findings stated above concerning the apical hook of peas lead us to assume that differential growth between the inner and outer sides of the plumular arc is controlled by some intrinsic properties probably of the plumule itself. The property-driven differential growth is not influenced by gravity until the hook is established, but is subsequently caused to sustain or enhance the hook (Figure 5). At the latter stage, where the hook has established and obtained responsiveness to gravity, if the seedling is turned upside down to apply gravity inversely, what will happen to the hook? This tempting question must be left to future studies.

Another example of morphogenesis by an intrinsic property in peas is the epicotyl bending which is found in cv. Alaska placed 
in space or on a clinostat (Ueda et al., 1999, 2000; Miyamoto et al., 2005a,b, 2007) or in ageotropum and described as automorphosis [Eigenrichtung by Pfeffer (1904); see the review by Stanković etal. (1998), Volkmann etal. (1986)]. The epicotyl bends about $40^{\circ}$ when it just starts to grow. This phenomenon may be explained as follows: an asymmetric growth of the epicotyl caused by some intrinsic property of the epicotyl takes place only for a limited time when the epicotyl starts to grow at the germination stage. In Alaska under $1-g$ conditions the intrinsic property is overcome by the influence of gravity or gravity-driven auxin transport and no epicotyl bending takes place.

Development of the apical hook is caused by differential elongation between the outer and inner sides of the plumular arc in the embryo, and has been reported to involve cell division and elongation (Raz and Koornneef, 2001), being controlled by various plant growth hormones including ethylene (Bleecker et al., 1988; Bleecker and Schaller, 1996; Lehman et al., 1996; Raz and Ecker, 1999; Vriezen et al., 2004; De Grauwe et al., 2005; Vandenbussche et al., 2010; Žádníková et al., 2010; Gallego-Bartolomé et al., 2011; Willige et al., 2012). Recent studies tend to indicate that these growth regulators exert their effects at the end through asymmetric distribution of auxin (Vandenbussche et al., 2010; Zádníková et al., 2010; Abbas et al., 2013). The present study showed that the apical hook formation was suppressed by auxin polar transport inhibitors, TIBA, NPA and HFCA, to almost the same extent in cv. Alaska as in ageotropum under $1-g$ conditions (Figure 8). The results suggest that polar transport of auxin distributed asymmetrically by the intrinsic property of the embryo plumule plays a role in the portion of the apical hook development which takes place independently of gravity. If the sustention or enhancement of the established hook by gravity (Figures 5 and 6) also involves auxin polar transport, it may readily be explained by possible downward translocation of auxin across the plumular axis, which has a horizontal portion (cf. Figure 2). That an apical hook requires higher auxin concentration at the inner than the outer side is an established knowledge (Abbas et al., 2013).

In a summary, time-course studies with normal Alaska pea and the agravitropic pea mutant, ageotropum, under $1-g$ conditions and on the 3-D clinostat revealed that (1) the apical hook of the epicotyl forms by development of the arc-shaped plumule of the embryo existing in the non-germinated seed. The process of formation consists of two stages: development and partial opening, and controlled by some intrinsic property of the plumule. Approximately when the epicotyl emerges from the seed, the hook is established in both pea varieties. In Alaska the established hook is sustained or enhanced by gravity, resulting in a delay of hook opening compared with on a clinostat, which might give an incorrect idea that gravity causes hook formation. Application of auxin polar transport inhibitors suppressed the curvature of hook in Alaska as well as in ageotropum, suggesting that the formation of the hook involves auxin polar transport independently of gravity action.

\section{ACKNOWLEDGMENT}

This study was partially supported by JSPS KAKENHI (Grant No. 1281205900 to Kensuke Miyamoto).

\section{REFERENCES}

Abbas, M., Alabadí, D., and Blázquez, M. A. (2013). Differential growth at the apical hook: all roads lead to auxin. Front. Plant Sci. 4:441. doi: 10.3389/fpls.2013.00441 Bleecker, A. B., Estelle, M. A., Somerville, C., and Kende, H. (1988). Insensitivity to ethylene conferred by a dominant mutation in Arabidopsis thaliana. Science 241, 1086-1089. doi: 10.1126/science.241.4869.1086

Bleecker, A. B., and Schaller, G. E. (1996). Update on signal transduction: the mechanism of ethylene perception. Plant Physiol. 111, 653-660.

De Grauwe, L., Vandenbussche, F., Tietz, O., Palme, K., and Van Der Straeten, D. (2005). Auxin, ethylene and brassinosteroids: tripartite control of growth in the Arabidopsis hypocotyl. Plant Cell Physiol. 46, 827-836. doi: 10.1093/pcp/ pcil11

Gallego-Bartolomé, J., Arana, M. V., Vandenbussche, F., Žádníková, P., Minguet, E. G., Guardiola, V., et al. (2011). Hierarchy of hormone action controlling apical hook development in Arabidopsis. Plant J. 67, 622-634. doi: 10.1111/j.1365313X.2011.04621.x

Halstead, T. W., and Dutcher, F. R. (1987). Plants in space. Annu. Rev. Plant Physiol. 38, 317-345. doi: 10.1146/annurev.pp.38.060187.001533

Hoson, T., Kamisaka, S., Buchen, B., Sievers, A., Yamashita, M., and Masuda, Y. (1996). Possible use of a 3-D clinostat to analyze plant growth processes under microgravity conditions. Adv. Sci. Res.17, 47-53.

Hoson, T., Kamisaka, S., Masuda, Y., Yamada, M., and Yamashita, M. (1988). Development of 3-D clinostat and its possible use for the analysis of plant growth processed. Proc. Space Util. Symp. 5, 52-56 (in Japanese with English abstract).

Hoson, T., Kamisaka, S., Masuda, Y., and Yamashita, M. (1992). Changes in plant growth processes under microgravity conditions simulated by a threedimensional clinostat. Bot. Mag. Tokyo 105, 53-70. doi: 10.1007/BF02489403

Hoson, T., Kamisaka, S., Masuda, Y., Yamashita, M., and Buchen, B. (1997). Evaluation of the three-dimensional clinostat as a simulator of weightlessness. Planta 203, S187-S197. doi: 10.1007/PL00008108

Hoson, T., and Soga, K. (2003). New aspects of gravity responses in plant cells. Int. Rev. Cytol. 229, 209-244. doi: 10.1016/S0074-7696(03)29005-7

Kraft, T. F. B., van Loon, J. J. W. A., and Kiss, J. Z. (2000). Plastid position in Arabidopsis columella cells is similar in microgravity and on a random-positioning machine. Planta 211, 415-422. doi: 10.1007/s004250000302

Lehman, A., Black, R., and Ecker, J. R. (1996). HOOKLESS1, an ethylene response gene, is required for differential cell elongation in the Arabidopsis hypocotyl. Cell 85, 183-194. doi: 10.1016/S0092-8674(00)81095-8

MacDonald, I. R., Gordon, D. C., Hart, J. W., and Maher, E. P. (1983). The positive hook: the role of gravity in the formation and opening of the apical hook. Planta 158, 76-81. doi: 10.1007/BF00395406

Miyamoto, K., Hoshino, T., Hitotsubashi, R., Yamashita, M., and Ueda, J. (2005a). Automorphosis-like growth in etiolated pea seedlings is induced by the application of chemicals affecting perception of gravistimulation and its signal transduction. Adv. Space Res. 36, 1263-1268. doi: 10.1016/j.asr.2005. 03.082

Miyamoto, K., Hoshino, T., Yamashita, M., and Ueda, J. (2005b). Automorphosis of etiolated pea seedlings in space is simulated by a three-dimensional clinostat and the application of inhibitors of auxin polar transport. Physiol. Plant. 123, 467-474. doi: 10.1111/j.1399-3054.2005.00472.x

Miyamoto, K., Hoshino, T., Takahashi, Y., and Ueda, J. (2007). Auxin polar transport of etiolated Ageotropum pea epicotyls is not affected by gravistimulation: relevance to automorphosis-like growth and development. Adv. Space Res. 39, 1140-1146. doi: 10.1016/j.asr.2007.02.087

Olsen, G. M., and Iversen, T.-H. (1980a). Growth and curvature in seedlings of Pisum sativum and an ageotropic mutant. Physiol. Plant. 50, 269-274. doi: 10.1111/j.1399-3054.1980.tb04462.x

Olsen, G. M., and Iversen, T.-H. (1980b). Ultrastructure and movements of cell structures in normal pea and an ageotropic mutant. Physiol. Plant. 50, 275-284. doi: 10.1111/j.1399-3054.1980.tb04463.x

Paul, A.-L., Wheeler, R. M., Levine, H. G., and Ferl, R. (2013). Fundamental plant biology enabled by the space shuttle. Am. J. Bot. 100, 226-234. doi: 10.3732/ajb. 1200338

Pfeffer, W. (1904). Pflanzenphysiologie, 2nd Edn. Leipzig: W. Engelmann.

Raz, V., and Ecker, J. R. (1999). Regulation of differential growth in the apical hook of Arabidopsis. Development 126, 3661-3668.

Raz, V., and Koornneef, M. (2001). Cell division activity during apical hook development. Plant Physiol. 125, 219-226. doi: 10.1104/pp.125.1.219 
Schöldéen, C., and Burström, H. (1960). Physiological studies of an ageotropic pea mutant. Physiol. Plant. 13, 831-838. doi: 10.1111/j.1399-3054.1960.tb 08106.x

Scott, I. M. (1988). Stem growth habit and starch statolish content of the creep pea mutant. J. Plant Physiol. 133, 103-106. doi: 10.1016/S0176-1617(88)80092-0

Shichijo, C., and Hashimoto, T. (2013). "Light-induced exaggeration of the hypocotyl hook: its developmental basis and significance," in From Germination to Young Plants, ed. C. A. Busso (New York: Nova Science Publishers, Inc.), 39-59.

Stanković, B., Volkmann, D., and Sack, F. D. (1998). Autotropism, automorphogenesis, and gravity. Physiol. Plant. 102, 328-335. doi: 10.1034/j.13993054.1998.1020222.x

Strudwick, N. J., Phillips, T. J., and Scott, I. M. (1997). Expression of the abnormal gravitropism phenotypes creep and ageotropum during development in pea. J. Plant Physiol. 150, 588-591. doi: 10.1016/S0176-1617(97) 80323-9

Takahashi, H., and Suge, H. (1988). Involvement of ethylene in gravity-regulated peg development in cucumber seedling. Plant Cell Physiol. 29, 313-320.

Taiz, L., and Zeiger, E. (2010). Plant Physiology, 5th Edn. Sunderland: Sinauer Associates, Inc.

Ueda, J., Miyamoto, K., Yuda, T., Hoshino, T., Fujii, S., Mukai, C., et al. (1999). Growth and development, and auxin polar transport in higher plants under microgravity conditions in space: BRIC-AUX on STS-95 space experiment. J. Plant Res. 112, 487-492. doi: 10.1007/PL00013904

Ueda, J., Miyamoto, K., Yuda, T., Hoshino, T., Sato, K., Fujii, S., et al. (2000). STS-95 space experiment for growth and development, and auxin polar transport. Biol. Sci. Space 14, 47-57. doi: 10.2187/bss.14.47

Vandenbussche, F., Petrášek, J., Žádníková, P., Hoyerová, K., Pesěk, B., Raz, V., et al. (2010). The auxin influx carriers AUX1 and LAX3 are involved in auxin-ethylene interactions during apical hook development in Arabidopsis thaliana seedlings. Development 137, 597-606. doi: 10.1242/dev.040790
Volkmann, D., Behrens, H. M., and Sievers, A. (1986). Development and gravity sensing of cress roots under microgravity. Naturwissenschaften 73, 438-441. doi: 10.1007/BF00367291

Vriezen, W. H., Achard, P., Harberd, N. P., and Van der Straeten, D. (2004). Ethylenemediated enhancement of apical hook formation in etiolated Arabidopsis thaliana seedlings is gibberellin dependent. Plant J. 37, 505-516. doi: 10.1046/j.1365313X.2003.01975.X

Willige, B. C., Ogiso-Tanaka, E., Zourelidou, M., and Schwechheimer, C. (2012). WAG2 represses apical hook opening downstream from gibberellin and PHYTOCHROME INTERACTING FACTOR 5. Development 139, 4020-4028.

Žádníková, P., Petrášek, J., Marhavý, P., Raz, V., Vandenbussche, F., Ding, Z., et al. (2010). Role of PIN-mediated auxin efflux in apical hook development of Arabidopsis thaliana. Development 137, 607-617. doi: 10.1242/dev.081240

Conflict of Interest Statement: The authors declare that the research was conducted in the absence of any commercial or financial relationships that could be construed as a potential conflict of interest.

Received: 28 October 2013; paper pending published: 13 November 2013; accepted: 22 March 2014; published online: 08 April 2014.

Citation: Miyamoto K, Yamasaki T, Uheda E and Ueda J (2014) Analysis of apical hook formation in Alaska pea with a 3-D clinostat and agravitropic mutant ageotropum. Front. Plant Sci. 5:137. doi: 10.3389/fpls.2014.00137

This article was submitted to Plant Physiology, a section of the journal Frontiers in Plant Science.

Copyright $(2014$ Miyamoto, Yamasaki, Uheda and Ueda. This is an open-access article distributed under the terms of the Creative Commons Attribution License (CC BY). The use, distribution or reproduction in other forums is permitted, provided the original author(s) or licensor are credited and that the original publication in this journal is cited, in accordance with accepted academic practice. No use, distribution or reproduction is permitted which does not comply with these terms. 